\title{
Inertia tensor as morphological descriptor for aggregation dynamics
}

\author{
Frédéric Gruy (*) \\ Ecole Nationale Supérieure des Mines, 158 cours Fauriel, 42023, Saint-Etienne, France \\ * corresponding author \\ gruy@emse.fr
}

0033477420202

\begin{abstract}
:
Multidimensional population balance modelling is a powerful tool to study the dynamics of the precipitation and particularly the aggregation of particles. This approach involves the selection of internal parameters describing the particle. Among them, morphological or geometrical descriptors are required. In this paper, the inertia or gyration tensor is considered and evaluated for the modelling of aggregation. An equivalent ellipsoid to the aggregate is defined from the tensor. Firstly, a general expression for the inertia tensor of the aggregate resulting from the collision of two aggregates is presented. In this framework, the collision event becomes the collision between two equivalent ellipsoids and leads to a larger ellipsoid. Secondly, the inertia tensor and the characteristics of the equivalent ellipsoid are explicitly calculated in the case of shear aggregation in a two-dimensional space. An approximate calculation is also presented and checked. Finally, the shear aggregation of a set of disks in a two-dimensional space is simulated using simultaneously this modelling of the collision event and Monte-Carlo simulations for all the collisions. This is compared with classical Monte Carlo simulations where the colliding particles are clusters of disks. Physical properties of the cluster population as, for instance, the distribution of elongation or anisotropy factor at different times are presented.
\end{abstract}


Keywords: aggregation, inertia tensor, anisotropy, equivalent ellipsoid, aggregation kernel

\section{Nomenclature}

$\vec{a} \quad$ vector between the centres of mass of two particles

a semi-major axis of ellipse

$b \quad$ semi-minor axis of ellipse

A anisotropy factor defined by [9-10]

$A_{i j} \quad$ anisotropy factor defined by [11]

B coefficient in Eq.35

$D_{f} \quad$ fractal dimension

$\underline{\underline{D_{E}}} \quad$ diagonal matrix for ellipsoid

$\underline{\underline{D_{I}}} \quad$ diagonalized inertia tensor

$\underline{D_{I, m}} \quad$ diagonalized inertia tensor after averaging

$\stackrel{I}{=} \quad$ inertia tensor of the particle

$k_{12} \quad$ contribution to $K_{12}$ (Eq.14)

$K_{12} \quad$ kernel of aggregation

$L \quad$ penetration length

M particle mass

$N \quad$ number of primary particles per aggregate

$P \quad$ configuration probability

$\vec{p} \quad$ orientation vector of ellipsoid

$R_{i} \quad$ square root of diagonal elements of $\underline{\underline{D_{I}}}$ 
$R_{g} \quad$ gyration radius

$U_{i} \quad$ semi-axis $i$ of equivalent ellipsoid

$V \quad$ particle volume

$\vec{v} \quad$ particle velocity

$\vec{x} \quad$ position vector of particle

$x, y, z: \quad$ space coordinates

Greek letters

$(\alpha, \beta, \gamma, \delta) \quad$ subscripts for configuration of two-ellipse set

$\varepsilon \quad$ porosity

$\phi \quad$ solid volume fraction or density

$\dot{\gamma} \quad$ shear rate

$\kappa \quad$ permeability

$\lambda$ elongation

$\rho \quad$ mass density

$\theta \quad$ orientation angle

Superscript

app approximate

(i) for tensor (particle $i$ )

Subscript

$\underline{\underline{X}} \quad$ tensor $X$

eq equivalent 
$i \quad$ for scalar or vector (particle $i)$

$i, k \quad k$ component of vector (particle $i$ )

$k, l \quad k, l$ element of the tensor or matrix

$m \quad$ lower integration boundary (Eq.15)

$M \quad$ upper integration boundary (Eq.15)

symbol

$<>\quad$ average

\section{Introduction}

Aggregation of particles in a suspension is due to the binary collisions between two particles. The resulting aggregate or cluster is a branched and porous object consisting of primary particles, usually considered as identical. Most often, the morphology of the cluster is described by using the fractal theory: it is defined by the radius of the primary particle, its number of primary particles and the fractal dimension. The last quantity is a real number within the 1-3 range. It is determined from simulations of colliding particles obeying a given collisional mechanism or from an experimental characterization tool as X-ray scattering (SAXS) device. Even if the fractal hypothesis was successful for describing the dynamics of aggregation, the fractal dimension is not the only morphological parameter to be relevant. The fractal dimension of stochastic aggregates is a statistical quantity; it either refers to a population of aggregates (as in SAXS analysis) or to a sufficiently large single aggregate (as in the analysis of microscope images); in addition fractal dimension is related to several morphological parameters (e.g. radius of gyration or mobility radius - when measured against aggregate mass, or interparticle distance (pair correlation) - when using box-counting or 
SAXS data). Moreover, the relation between its value and the nature of the collisional mechanism is not so obvious.

A convex particle, as a spherical primary particle, or a non-convex one, as an aggregate, may be described by several morphological parameters, the relevance of which depending on the considered physical phenomenon. These parameters can be organized as a vector or as a tensor. The theory of scalar or Tensorial Minkowski Functionals (TMF) provides the rigorous framework for such parameter set. Mecke et al. [1,2] have applied the integral geometry in various fields of physics. They underlined that the inertia tensor does not belong to the TMF because it does not possess all the mathematical properties. However, inertia tensor is similar to TMF. Porosity, anisotropy and orientation of an object are geometrical and textural characteristics that investigators have frequently considered. Inertia tensor seems an interesting candidate for introducing these quantities. This is a more popular concept among physicists than the Minkowski functionals.

Torres et al. [3] have, for instance, performed simulations of 3-D shear aggregation. They found a fractal dimension value equal to 1.83 and they observed the non spherical shape of the aggregate. Moreover they have calculated the inertia tensor (normalized by the square of the gyration radius) for clusters with a number $N$ of primary particles smaller than 4100 . They have observed that the semi-axes of the equivalent ellipsoid were in the ratio 1/1.6/3.1 whatever the origin of the particle collision (Brownian motion, shear flow, elongation flow).

Investigators defined two quantities: inertia tensor [4] and gyration tensor [5]. The first one considers the mass and the position vector distributions inside the particle. The second one that has, in fact, the same definition is often used for systems, polymers or aggregates, consisting in monomers or identical primary particles; in this case it only depends on the position vector distribution. The words "inertia tensor" will be used later on in this paper. 
One considers a set of $N$ points with Cartesian coordinates $x_{k}, y_{k}, z_{k}$ or $x_{k, 1}, x_{k, 2}, x_{k, 3}$ and a mass equal to one in a three-dimensional space. The origin of the coordinate system is the centre of mass. The inertia tensor is defined by the expression [4-8]:

$\underline{I}=\sum_{k=1}^{N}\left[\begin{array}{ccc}x_{k, 2}^{2}+x_{k, 3}^{2} & -x_{k, 1} x_{k, 2} & -x_{k, 1} x_{k, 3} \\ & x_{k, 1}^{2}+x_{k, 3}^{2} & -x_{k, 2} x_{k, 3} \\ & & x_{k, 1}^{2}+x_{k, 2}^{2}\end{array}\right]$

or

$I_{i, j}=\sum_{k=1}^{N}-x_{k, i} x_{k, j}+\delta_{i j} \vec{x}_{k}^{2}$

The continuous formulation of the tensor component is:

$I_{i, j}=\int_{[V]}\left(-x_{i} x_{j}+\delta_{i j} \vec{x}^{2}\right) d V$

The diagonalization of $\underline{I}$ leads to:

$\underline{\underline{D_{I}}}=\left[\begin{array}{ccc}R_{1}^{2} & 0 & 0 \\ 0 & R_{2}^{2} & 0 \\ 0 & 0 & R_{3}^{2}\end{array}\right]$

with $R_{1}<R_{2}<R_{3}$

$R_{1}^{2}, R_{2}^{2}$ and $R_{3}^{2}$ are the principal moments of inertia (divided by the particle mass).

Then, the gyration radius and the anisotropy factor are defined as:

$R_{g}^{2}=R_{1}^{2}+R_{2}^{2}+R_{3}^{2}$

$A_{i j}=R_{i}^{2} / R_{j}^{2}$

Otherwise Rudnick and Gaspari [9-10] define the anisotropy factor also named the asphericity by:

$$
A=\frac{\left(R_{1}^{2}-R_{2}^{2}\right)^{2}+\left(R_{1}^{2}-R_{3}^{2}\right)^{2}+\left(R_{2}^{2}-R_{3}^{2}\right)^{2}}{2\left(R_{1}^{2}+R_{2}^{2}+R_{3}^{2}\right)^{2}}
$$


$R_{g}$ and $A$ are rotational invariants.

Pranami [11] et al. have built by simulation a set of clusters and have shown that the fractal dimension $D_{f}$ is not the only relevant parameter; the two anisotropy factors $A_{31}$ and $A_{21}$ have to be considered. Researchers had previously considered fractal-like clusters with spherical symmetry defined by the relation between the gyration radius and the number of primary particles or their mass. The corresponding distribution functions of the two anisotropy factors in the population are very close whatever the number of primary particles in the clusters. As a consequence it seems that a cluster may be described as a spheroid rather than an ellipsoid. The authors indicate that the fractal dimension is qualitatively related to the anisotropy distribution: as $D_{f}$ value increases, the mean anisotropy factor decreases.

Thus the inertia tensor and the mass are interesting candidates for describing a particle (particle is understood as a general term). If the orientation of the particle is not required, the knowledge of the three eigenvalues of the inertia tensor and of the mass or the matter volume is sufficient. Then one can deduce the porosity, itself defined from the gyration radius, and the anisotropy factors.

Starting from this idea, we will study in this paper the change of inertia tensor and mass during a collision and an aggregation of two particles or clusters. We will also analyse the consequences on the entire aggregation dynamics.

Section 2 presents the theoretical elements corresponding to the collision of two clusters. Section 3 is dedicated to its application in a two-dimensional space. Section 4 contains the results for the aggregation dynamics of the entire population. Section 5 concludes the paper.

\section{Change of inertia tensor during the collision of two particles}




\subsection{Theoretical background}

The aggregation dynamics of a suspension is studied by means of a population balance, the particles being described by their internal parameters. The morphological parameters are among these. The additivity of the parameters of two colliding particles, denoted 1 and 2 , is an important property needed for a concise writing of the population balance. This property is verified by the mass or the matter volume:

$$
M_{1+2}=M_{1}+M_{2}
$$

This is almost the case for the inertia tensor if it is evaluated about the centre of mass of the resulting cluster. This will appear in this section.

Let us consider two objects with the inertia tensors $\underline{\underline{I}}^{(1)}$ and $\underline{\underline{I}}^{(2)}$, each one evaluated about its own centre of mass $C_{i}$. The inertia tensor evaluated about another point $C_{i}{ }^{\prime}$ (the vector $\vec{a}$ is defined by $\vec{a}=\overrightarrow{C_{i}{ }^{\prime} C_{i}}$ ) becomes [12] :

$$
I_{k l}^{(i)}{ }^{\prime}=I_{k l}^{(i)}+M_{i}\left(\vec{a}^{2} \delta_{k l}-a_{k} a_{l}\right) \quad i=1,2
$$

Then the inertia tensor of the object resulting from the collision of two smaller objects with the centres of mass separated by $\vec{a}=\overrightarrow{C_{1} C_{2}}$ obeys the equation:

$$
I_{k l}^{(1+2)}=I_{k l}^{(1)}+I_{k l}^{(2)}+\frac{M_{1} M_{2}}{M_{1}+M_{2}}\left(\vec{a}^{2} \delta_{k l}-a_{k} a_{l}\right)
$$

One defines the equivalent ellipsoid as the real ellipsoid having the same diagonalized inertia tensor as the object $(1,2$ or $1+2)$. Each equivalent ellipsoid has an orientation defined by the vector $\overrightarrow{p_{i}}$. The solid volume of primary particles is smaller than the geometrical volume of the equivalent ellipsoid. I assume that the vector $\vec{a}=\overrightarrow{C_{1} C_{2}}$ is such as the equivalent ellipsoids of the two colliding clusters are tangent in the sense of the geometry (figure 1). This 
hypothesis is not trivial and will be discussed later on: as the radius of gyration of fractal aggregates is smaller than the geometrical size, one may expect that the distance between the centres of the two colliding equivalent ellipses will be shorter than the previously assumed one.

The equivalent ellipsoid of the resulting object is obtained from the diagonalization of the tensor $\underline{I}^{(1+2)}$; the corresponding tensor is denoted $\underline{D_{I}^{(1+2)}}$ (see Eq.3). The equivalent ellipsoid with semi-axes $\left(U_{1}, U_{2}, U_{3}\right)$ is such as:

$$
\begin{aligned}
& U_{1}^{2}=\frac{5}{2}\left(R_{2}^{2}+R_{3}^{2}-R_{1}^{2}\right) \\
& U_{2}^{2}=\frac{5}{2}\left(R_{1}^{2}+R_{3}^{2}-R_{2}^{2}\right) \\
& U_{3}^{2}=\frac{5}{2}\left(R_{1}^{2}+R_{2}^{2}-R_{3}^{2}\right)
\end{aligned}
$$

One will introduce the tensor $\underline{\underline{D_{E}}}$ which contains all the geometrical parameters of the equivalent ellipsoid:

$$
\underline{\underline{D_{E}}}=\left[\begin{array}{ccc}
U_{1}^{2} & 0 & 0 \\
0 & U_{2}^{2} & 0 \\
0 & 0 & U_{3}^{2}
\end{array}\right]
$$

Then, the mathematical treatment of a collision event of two clusters, which are approximated as ellipsoids, will follow the sequence:

1. transform ellipsoid to inertia tensors for defined orientation:

$$
\underline{\underline{D_{E}^{(i)}}} \rightarrow \underline{\underline{D_{I}^{(i)}}} \stackrel{\overrightarrow{p_{i}}}{\longrightarrow} \underline{\underline{I^{(i)}}} \quad \text { with } i=1,2
$$

2. compute new inertia tensor:

$$
\underline{\underline{I^{(1)}}}+\underline{\underline{I^{(2)}}} \rightarrow \underline{\underline{I^{(1+2)}}}
$$

3. determine the geometric properties of the equivalent ellipsoid:

$$
\underline{\underline{I^{(1+2)}}} \rightarrow \underline{\underline{D_{I}^{(1+2)}}} \rightarrow \underline{\underline{D_{E}^{(1+2)}}}
$$


In practice, aggregation involves a large number of particles; a sub-set of particles has the same morphological characteristics, but with different orientations. So the description of a collision between two objects with different morphological parameters needs the average over all the possible orientations of the two objects $\left\langle\underline{\underline{D^{(1+2)}}}\right\rangle_{(p 1, p 2)}$. The mean quantities \langle\rangle$_{p i}$ are performed over all the orientations. The average is applied to $\underline{\underline{D_{I}^{(1+2)}}}$. This procedure has been selected for the following reason:

Specifying the orientation of the ellipsoids for each collision add a unit vector, i.e. its components, into the set of internal variables describing the particle. In order to reduce the number of internal variables an averaging over orientations and relative positions is done. Hence a particle is characterized by only its mass and the lengths of the ellipsoid semi-axes. This will be particularly important when solving the corresponding population balance equation.

The orientations of the two colliding objects are assumed non-correlated, i.e. the hydrodynamic resistance between two particles moving towards each other is not taken into account. The orientation angle distribution of a particle in a fluid depends on its shape and on the flow field. The relative position of the centres of mass at impact $\vec{a}=\overrightarrow{C_{1} C_{2}}$ depends both on the orientations of the two objects and on the mechanism of collision. The figure 2 includes some possible events. Finally the inertia tensor of the resulting cluster after the collision of two smaller clusters is:

$$
\underline{\underline{D_{I, m}^{(1+2)}}}=\left\langle\left\langle\underline{\underline{D_{I}^{(1+2)}}}\right\rangle_{(C 1, C 2)}\right\rangle_{(p 1, p 2)}
$$

The two main mechanisms, so-called perikinetic and orthokinetic, for aggregation are characterized by different kinetic constants or kernels. From a geometrical point of view: 
- In the case of perikinetic aggregation, two particles moving thanks to Brownian motion have the same collision probability whatever the relative angular position.

- In the case of orthokinetic aggregation, the probability of encounter is all the higher as the two particles are offset to the side in the shear flow. The impact of particles moving on the same streamline is unlikely.

Thus, one may write:

$\underline{\underline{D_{I, m}^{(1+2)}}}=\left\langle\left\langle\underline{\underline{D_{I}^{(1+2)}}}\right\rangle_{(C 1, C 2)}\right\rangle_{(p 1, p 2)}=\left\langle\int_{\Sigma(p 1, p 2)} \underline{\underline{D_{I}^{(1+2)}}} d k_{12}\right\rangle_{(p 1, p 2)} / K_{12}$

$K_{12}$ is the kernel as usual. $d k_{12}$ is the contribution of an infinitesimal interception or collision area to the kernel. One performs the sum over all the collision area. The whole collision area, that depends on the orientations of the two particles, is denoted by $\Sigma(p 1, p 2)$

As a consequence, the kernel obeys the relation:

$\left\langle\int_{\Sigma(p 1, p 2)} d k_{12}\right\rangle_{(p 1, p 2)}=K_{12}$

This approach will be applied to the case of the orthokinetic aggregation.

\subsection{Application in the case of aggregation under shear flow}

One considers a shear flow in a three-dimensional space (figure 3): the coordinate system is such as the velocity vector of the fluid carrying the particle is $\left(v_{x}, v_{y}, v_{z}\right)=(\dot{\gamma} y, 0,0) \cdot \dot{\gamma}$ is the shear rate.

A preliminary study concerns the orientation distribution of a single particle, as an equivalent ellipsoid, in the shear flow. The particle undergoes both translation and rotation. 
Considering a prolate spheroidal particle, its orientation is defined thanks to the vector $\vec{p}$ that lies along the major axis of the spheroid. The dynamics of the spheroidal particle in any flow has been extensively studied [13-14]. In a shear flow, the end of the vector $\vec{p}$ follows circular (and periodic) trajectories called Jeffery trajectories [15]. The characteristics of these trajectories are not sensitive to the environment of the particle like walls or other particle. If the particles are non-spheroidal ellipsoids, the motion becomes biperiodic and is very sensitive to the deviation from axisymmetry [16]. One may conclude that an ellipsoid has no preferred orientation in a shear flow and that, as a rough approximation, the orientation angle distribution of a single ellipsoid is uniform.

Moreover, the hydrodynamic resistance [17-19] will be neglected in the paper.

Thus, one may calculate the diagonal inertia tensor after averaging over the centre of mass position and orientation:

$\underline{\underline{D_{I, m}^{(1+2)}}}=\left\langle\int_{Y m(p 1, p 2)}^{Y M(p 1, p 2)} \int_{Z m(y, p 1, p 2)}^{Z M(y, p 1, p 2)} \underline{\underline{I}}^{(1+2)} \dot{\gamma} y d z d y\right\rangle_{(p 1, p 2)} / K_{12}$

with

$$
K_{12}=\left\langle\int_{Y m(p 1, p 2)}^{Y M(p 1, p 2)} \int_{Z m(y, p 1, p 2)}^{Z M(y, p 1, p 2)} \dot{\gamma} y d z d y\right\rangle_{(p 1, p 2)}
$$

$Z_{m}, Z_{M}, Y_{m}$ and $Y_{M}$ are the integration boundaries: there is no collision event beyond these values of the coordinates of the centres of mass.

The above theory and the developed methodology will be applied to the two-dimensional problem, i.e. the aggregation of ellipses moving in a two-dimensional shear flow.

\section{Aggregation of ellipses in a two-dimensional shear flow}


The collision of two ellipses 1 and 2 is studied in a two-dimensional space (x,y); The flow velocity vector is given by $\left(v_{x}, v_{y}\right)=(\dot{\gamma} y, 0)$.

The diagonalized inertia tensor of the resulting cluster becomes:

$\underline{\underline{D_{I, m}^{(1+2)}}}=\left\langle\int_{Y m(p 1, p 2)}^{Y M(p 1, p 2)}{\underline{\underline{D^{(1+2)}}}}_{\bar{\gamma}} y d y\right\rangle_{(p 1, p 2)} / K_{12}$

with

$$
K_{12}=\left\langle\int_{Y m(p 1, p 2)}^{Y M(p 1, p 2)} \dot{\gamma} y d y\right\rangle_{(p 1, p 2)}
$$

The inertia tensor of an ellipse with semi-axes $a$ and $b(a>b)$ and with the semi-major axis inclined by $\theta$ angle with $\mathrm{x}$ axis is given by:

$$
\underline{I}=\int_{[S]} \rho\left[\begin{array}{cc}
x^{2} & -x y \\
-x y & y^{2}
\end{array}\right] d x d y=\rho \frac{\pi}{4}(a b)^{3}\left[\begin{array}{cc}
(\sin \theta / a)^{2}+(\cos \theta / b)^{2} & \sin \theta \cos \theta\left(a^{-2}-b^{-2}\right) \\
\sin \theta \cos \theta\left(a^{-2}-b^{-2}\right) & (\sin \theta / b)^{2}+(\cos \theta / a)^{2}
\end{array}\right]
$$

After performing the diagonalization:

$$
\underline{\underline{D_{I}}}=\rho \frac{\pi}{4}(a b)^{3}\left[\begin{array}{cc}
b^{-2} & 0 \\
0 & a^{-2}
\end{array}\right]=\left[\begin{array}{cc}
M a^{2} / 4 & 0 \\
0 & M b^{2} / 4
\end{array}\right]
$$

with $\quad M=\rho \pi a b$

$\rho$ and $M$ are the 2-D density and the mass of the interior of the ellipse.

The steps of the calculation are the following ones:

- Step 1: fix the orientation vectors for the two ellipses $\overrightarrow{p_{1}}, \overrightarrow{p_{2}}$

- Step 2 : fix the y-coordinate of the centre of mass of the ellipse 2

- Step 3: calculate the coordinates of the intersection point (collision impact) of the two ellipses 
- Step 4: deduce the coordinates of the vector $\vec{a}$ (between the centres of mass of the two ellipses)

- Step 5: calculate $\underline{\underline{I^{(1+2)}}}$, then $\underline{\underline{D_{I}^{(1+2)}}}$ and $K_{12}$

- Step 6: go to step 1 for a new configuration of the two ellipses

- Step 7: perform the mean values of $I^{(1+2)}, D_{I}^{(1+2)}$ and $K_{12}$ over all the configurations The two elements of $\underline{\underline{D_{I}^{(1+2)}}}$, i.e. the eigenvalues of $\underline{\underline{I}}$, are ordered such as $D_{11}^{(1+2)}>D_{22}^{(1+2)}$.

The kernel of the two-dimensional shear aggregation is obtained following the work of Von Smoluchowski [20] who considered the collision of two spheres. The contribution to the kernel due to two colliding ellipses with given orientations ( $\theta_{1}$ and $\theta_{2}$ angles) and parameters $\left(a_{1}, b_{1}, a_{2}, b_{2}\right)$ is:

$k_{12}\left(\theta_{1}, \theta_{2}\right)=2 \int_{0}^{y_{12, \max }} \dot{\gamma} y d y=\dot{\gamma} y_{12, \max }^{2}$

with

$$
y_{12, \max }=\sqrt{\left(a_{1} \sin \theta_{1}\right)^{2}+\left(b_{1} \cos \theta_{1}\right)^{2}}+\sqrt{\left(a_{2} \sin \theta_{2}\right)^{2}+\left(b_{2} \cos \theta_{2}\right)^{2}}
$$

By averaging $k_{12}$ over all the orientations of the two ellipses:

$$
K_{12}=\left\langle k_{12}\right\rangle_{p 1, p 2}=\frac{\dot{\gamma}}{\pi^{2}} \int_{0}^{\pi} \int_{0}^{\pi} y_{12, \max }^{2} d \theta_{1} d \theta_{2}
$$

Then,

$$
K_{12}=\dot{\gamma}\left[\frac{a_{1}^{2}+b_{1}^{2}}{2}+\frac{a_{2}^{2}+b_{2}^{2}}{2}+\frac{8 a_{1} a_{2}}{\pi^{2}} E\left(1-\left(b_{1} / a_{1}\right)^{2}\right) E\left(1-\left(b_{2} / a_{2}\right)^{2}\right)\right]
$$

where $E(m)$ is the complete elliptic integral of the second kind.

This kernel has to be compared to the one of two circular disks having the same areas that the ellipses: 


$$
K_{12}^{\text {disks }}=\dot{\gamma}\left[\left(a_{1} b_{1}\right)^{1 / 2}+\left(a_{2} b_{2}\right)^{1 / 2}\right]^{2}
$$

The calculation of the intersection point (step 3) is not trivial and needs some computational time. This feature will be particularly critical when studying the tri-dimensional collision modelling. As the previous algorithm will be included in a larger program for solving the population balance, we have also considered an approximation of the whole sequence (steps 1-7) in order to reduce the computational time:

- The continuous variables and functions like the distribution of orientations and the relative position of ellipses are replaced by a limited number of configurations.

- Four simple configurations (denoted $\mathrm{q}=\alpha, \beta, \gamma, \delta$ ) are selected so that the calculation of their inertia tensor $\underline{\underline{I_{q}}}$ is easy (figure 4).

- A probability $P_{q}$ proportional to the y-coordinate of the vector $\vec{a}=\overrightarrow{C_{1} C_{2}}$ is given for each configuration:

$$
\begin{aligned}
& \underline{\underline{I_{\alpha}}}=\left[\begin{array}{cc}
\sup \left(u_{\alpha}, v_{\alpha}\right) & 0 \\
0 & \inf \left(u_{\alpha}, v_{\alpha}\right)
\end{array}\right] \\
& P_{\alpha}=\left(b_{1}+b_{2}\right) / 2 /\left(a_{1}+a_{2}+b_{1}+b_{2}\right) \\
& u_{\alpha}=M_{1} a_{1}^{2} / 4+M_{2} a_{2}^{2} / 4+M_{1} M_{2} /\left(M_{1}+M_{2}\right)\left(b_{1}+b_{2}\right)^{2} \\
& v_{\alpha}=M_{1} b_{1}^{2} / 4+M_{2} b_{2}^{2} / 4
\end{aligned}
$$

$\underline{\underline{I_{\beta}}}=\left[\begin{array}{cc}\sup \left(u_{\beta}, v_{\beta}\right) & 0 \\ 0 & \inf \left(u_{\beta}, v_{\beta}\right)\end{array}\right]$

$P_{\beta}=\left(b_{1}+a_{2}\right) / 2 /\left(a_{1}+a_{2}+b_{1}+b_{2}\right)$

$u_{\beta}=M_{1} a_{1}^{2} / 4+M_{2} b_{2}^{2} / 4+M_{1} M_{2} /\left(M_{1}+M_{2}\right)\left(b_{1}+a_{2}\right)^{2}$

$v_{\beta}=M_{1} b_{1}^{2} / 4+M_{2} a_{2}^{2} / 4$ 


$$
\begin{aligned}
& \stackrel{I_{\gamma}}{=}=\left[\begin{array}{cc}
\sup \left(u_{\gamma}, v_{\gamma}\right) & 0 \\
0 & \inf \left(u_{\gamma}, v_{\gamma}\right)
\end{array}\right] \\
& P_{\gamma}=\left(a_{1}+b_{2}\right) / 2 /\left(a_{1}+a_{2}+b_{1}+b_{2}\right) \\
& u_{\gamma}=M_{1} b_{1}^{2} / 4+M_{2} a_{2}^{2} / 4+M_{1} M_{2} /\left(M_{1}+M_{2}\right)\left(a_{1}+b_{2}\right)^{2} \\
& v_{\gamma}=M_{1} a_{1}^{2} / 4+M_{2} b_{2}^{2} / 4 \\
& \underline{\underline{I_{\delta}}}=\left[\begin{array}{cc}
\sup \left(u_{\delta}, v_{\delta}\right) & 0 \\
0 & \inf \left(u_{\delta}, v_{\delta}\right)
\end{array}\right] \\
& P_{\delta}=\left(a_{1}+a_{2}\right) / 2 /\left(a_{1}+a_{2}+b_{1}+b_{2}\right) \\
& u_{\delta}=M_{1} b_{1}^{2} / 4+M_{2} b_{2}^{2} / 4+M_{1} M_{2} /\left(M_{1}+M_{2}\right)\left(a_{1}+a_{2}\right)^{2} \\
& v_{\delta}=M_{1} a_{1}^{2} / 4+M_{2} a_{2}^{2} / 4 \\
& \underline{\underline{I^{a p p}}}=\underline{\underline{I_{\alpha}}} \underline{\underline{\underline{I_{\beta}}}} \underline{\underline{\underline{I_{\beta}}}} \underline{\underline{I_{\gamma}}} \underline{\underline{P_{\gamma}}}+\underline{\underline{I_{\delta}}} P_{\delta}
\end{aligned}
$$

By construction, the tensor $\underline{\underline{I^{a p p}}}$ is diagonal. The parameters $\left(a_{e q}, b_{e q}\right)$ of the equivalent ellipse of the resulting cluster are deduced from:

$$
\underline{\underline{I^{a p p}}}=\left[\begin{array}{cc}
\left(M_{1}+M_{2}\right) a_{e q}^{2} / 4 & 0 \\
0 & \left(M_{1}+M_{2}\right) b_{e q}^{2} / 4
\end{array}\right] \text { with } \quad a_{e q}>b_{e q}
$$

The accuracy of the approximate method (Eqs.27-28) has been compared to the exact method (Eqs.17-20): $\left(a_{e q}, b_{e q}\right)$ have been calculated from $\underline{\underline{I^{a p p}}}$ and from $\underline{\underline{D_{I, m}}}$ :

- Firstly the values of $\left(a_{e q}, b_{e q}\right)$ coming from collisions of identical ellipses in an aggregation step $(\mathrm{N})+(\mathrm{N}) \rightarrow(2 \mathrm{~N})$ were considered. The deviation between the two methods is smaller than $5 \%$ as the elongation, defined as $a_{e q} / b_{e q}$, is smaller than 10 . The good performance of the approximate method may be related to the fact that in all four configurations the systems of principal axes were either in parallel or perpendicular, and that always one pair of principal axes is on one line. Hence the calculation is performed on diagonal tensors. 
- Then the collisions between two differently sized ellipses were considered. The approximate method does not agree with the exact method if the ellipses have very different masses. As a consequence, the approximate method has to be corrected: the parameters of the resulting ellipse will be the one of the ellipse with the larger mass if the ratio between the two masses is larger than 30. By applying this correction, the error is smaller than $1 \%$ (respectively $5 \%$ ) in $50 \%$ (respectively $90 \%$ ) of the collision events.

\section{Results and discussion}

One considers an initial monodisperse population of disks (with radius 1 and with density 1). The aggregation of this two-dimensional suspension is studied. In our case, aggregation is a series of collisions between equivalent ellipses; each collision event corresponds to:

$\left(M_{1}, D_{11}^{(1)}, D_{22}^{(1)}\right)+\left(M_{2}, D_{11}^{(2)}, D_{22}^{(2)}\right) \stackrel{K_{12}}{\longrightarrow}\left(M_{1}+M_{2}, D_{11}^{(1+2)}, D_{22}^{(1+2)}\right)$

The subscript $m$ is withdrawn from the diagonal elements of $\underline{D_{I, m}}$, considering that the equivalent ellipse has forgotten the memory of its construction.

The collision event may be rewritten as:

$\left(M_{1}, \lambda_{1}, \varepsilon_{1}\right)+\left(M_{2}, \lambda_{2}, \varepsilon_{2}\right) \stackrel{K_{12}}{\longrightarrow}\left(M_{1}+M_{2}, \lambda_{1+2}, \varepsilon_{1+2}\right)$

where $\lambda$ and $\varepsilon$ are the elongation and the porosity of the object. The elongation is the ratio of the major semi-axis over the minor semi-axis $(\lambda>1)$ :

$\lambda=\sqrt{D_{11} / D_{22}}$

The mass density $\phi=1-\varepsilon$ is the ratio of the $N$ disk areas over the ellipse area:

$\phi=\pi N^{2} / 4 \sqrt{D_{11} D_{22}}$ 
The hierarchical aggregation and the polydisperse aggregation will be successively examined.

\subsection{Hierarchical aggregation}

The sequence of the collision events is the following (written as the number $N$ of primary particles in the aggregate):

$1+1 \rightarrow 2 ; 2+2 \rightarrow 4 ; 4+4 \rightarrow 8 ; 8+8 \rightarrow 16 ; \ldots$.

The figures 5-6 represent respectively $\lambda(N)$ and $\phi(N)$. One observes that these two quantities follow the power laws:

$\phi=1.26 N^{-0.55}$

$a_{N} / R_{1}=0.606 N^{0.78}$

$\lambda \rightarrow 2.16$

$R_{l}$ and $a_{N}$ are respectively the radius of the primary particle and the major semi-axis of the equivalent ellipse for the cluster with $N$ primary particles.

The elongation factor rapidly converges to a constant value. The mass density in the ellipse corresponds to a fractal object with a fractal dimension $D_{f}$ equal to 1.29 . The fractal dimension is calculated from the relation:

$\phi=S^{2 / D f} N^{(D f-2) / D f}$

if $a_{N} / R_{1}=(N / S)^{1 / D f}$ where $S$ is a structure factor.

The simulations of the hierarchical aggregation by using the approximate method are in good agreement ( $\left.\lambda=2.14, \phi=1.4 N^{-0.65}\right)$ with the ones obtained with the exact method.

\subsection{Polydisperse aggregation}


In this case, all the collision events are possible (for instance, $(3)+(6) \rightarrow(9)$ ). One starts from a monodisperse set of disks (with radius 1). A Monte-Carlo Simulation (MCS) is performed: The probability to have a collision between any two particles is proportional to the corresponding kinetic constant (kernel) of aggregation. Hence, two objects are randomly selected by applying this probability distribution. Then its equivalent ellipse is calculated. The process is repeated until clusters with a large number $\left(N=10^{4}\right)$ of primary particles are built. The chosen procedure for MCS was developed by Smith and Matsoukas [21]. At a given time, the population of clusters, i.e. equivalent ellipses, can be analysed: distribution of $N$, distribution of elongation, fractal dimension ....

The simulations obtained by the approximate (Eqs.27-28) and exact (Eqs.17-20) methods are in agreement (figure 7). With the approximate method, a power law is observed: $\phi=1.1 N^{-0.59}$ and the elongation is distributed within the range [1.0-2.25] (figure 8), whereas, with the exact method, the power law is $\phi=1.1 N^{-0.53}$ (figure 9) and the elongation is distributed within the range [1.3-2.25] (figure 10a). The last result is close to the one of Pranami ([11] figure 3). The figure $10 \mathrm{~b}$ has the same content of the figure 10a but with the presentation of Pranami. An asymptotic state for the elongation distribution is early reached $(\mathrm{t}<20)$. The fractal dimension deduced from $\phi(N)$ is close to 1.31 .

The literature contains several results concerning the shear aggregation in a 2-D space:

- Some authors consider shear aggregation as a ballistic aggregation. However, other authors dispute this point (see Elimelech et al. ([22], p.182))

- Hentschel [23] recalls that Meakin showed that $D_{f}=1.55$ for 2-D ballistic clustercluster aggregation. He adds that the anisotropy factor (as the ratio of the two largest principal gyration radii) is equal to 2 . From dimensional arguments, Hentschel finds $D_{f}=1.375$; This value does not depend on the collision mechanism and is consistent 
with a very weak interpenetration of clusters $L \square R^{2-D f}=R^{0.625}$ and an anisotropy factor equal to 2 .

- Meakin [24] performed cluster-cluster simulations in a 2-D space: the value of the fractal dimension is $1.50\left(D_{f}\right.$ increases as $N$ is increasing and is close to 1.55 when $\left.100<N<10^{4}\right)$.

It is possible that the deviation between our value of the fractal dimension and the value of Meakin (for instance) is due to the interpenetration of the equivalent ellipses. Thus the following improvement is proposed:

The equivalent ellipse consists in a hard core and a penetrable shell with the thickness depending on the permeability $\kappa$ of the ellipse. The contact point between the two colliding ellipses is at the level of the two core boundaries. Several authors evaluate the penetration length of a cluster (see Potanin [14], Veerapaneni et al. [25], Neale et al. [26]). They agree by proposing the relation $L=2 \sqrt{\kappa}$ (valid if the fractal dimension is higher than 1.8 in a $3-\mathrm{D}$ space). The permeability of a set of disks or of a bundle of parallel cylinders with radius $\mathrm{R}_{1}$ has been calculated following Sangani and Acrivos [27]:

$$
\begin{aligned}
& \kappa=R_{1}^{2}\left[-0.5 \ln \phi-0.738+\phi-0.887 \phi^{2}+2.038 \phi^{3}\right] /(4 \phi) \quad \phi<0.28 \\
& \kappa=R_{1}^{2} 2^{3 / 2}\left[1-(4 \phi / \pi)^{1 / 2}\right]^{5 / 2} /(9 \phi) \quad \phi>0.28
\end{aligned}
$$

$\phi$ is the volume fraction of cylinders.

Then the distance $\left(C_{1} C_{2}\right)$ between the centres of the two equivalent ellipses is reduced due to the reciprocal penetration of the two equivalent ellipses:

$$
\left(C_{1} C_{2}\right)=\left(C_{1} C_{2}\right)_{0}-B\left(\sqrt{\kappa_{1}}+\sqrt{\kappa_{2}}\right)
$$

$B$ is a proportionality coefficient close to 2 . The permeability $\kappa$ is given by the equation 34 .

Other expressions about the dependence of $\left(C_{1} C_{2}\right)$ with $\sqrt{\kappa}$ lead to similar results. The interpenetration leads to a densification that is implicitly handled by the model. 
As the model described in the paper is not based on the concept of fractality, and thus does not consider explicitly the inhomogeneous density inside the object, the permeability is calculated for a porous medium with a constant density. The latter one is the mean value of density inside the aggregate. This option is chosen because it is consistent along the paper. However the permeability (as a function of the density) should be calculated for the density valued at the surface of the fractal object. The mean value of the density in the aggregate is larger than the density value at the boundary of the aggregate. This is a limitation of the model.

Simulations have been performed with a corrected inter-centre distance (Eq.35). Several $B$ values have been tested. Whatever the $B$ value, the density versus $N$ follows a power law. However, the function $\phi(N)$ has two expressions depending on the number of primary particles into the aggregate. The corresponding results are gathered in the table 1 for calculations with the approximate method, and in the table 2 for calculations with the exact method. As expected the fractal dimension increases if the $B$ parameter increases. This is particularly verified for the smallest aggregates.

Monte Carlo simulations have been performed where the collision of two equivalent ellipses is replaced by the collision of two clusters of primary particles (with radius equal to one). The algorithm structure is the same as the one used for studying the dynamics of the population of equivalent ellipses. However the details of the step of collision are slightly different. The clusters are randomly oriented with a fixed orientation whereas the relative position (perpendicular to the streamline) of the two particles is fixed as well. The centre of mass of the cluster 1 is located at the origin of the coordinate system whereas the one of the cluster 2 is computationally made to move along a streamline in the shear flow. When two primary particles belonged to two different clusters collide, the resulting cluster is a new one in the population. The restructuring of the aggregate is not taken into account. So an aggregate is 
completely defined by the set of the Cartesian coordinates of all the primary particle centres. The number $N$ of primary particles per aggregate, the gyration radius $R_{g}$ and the elongation $(a / b)$ of the aggregate for all the cluster set are recorded at several times during a simulation run. The kernel, needed in the MCS, obeys the relation:

$$
K_{12}=2 \dot{\gamma}\left[R_{g, 1}+R_{g, 2}\right]^{2}
$$

This equation is consistent with the expression (24) valid for the collision between two ellipses with the gyration radii $R_{g, i}=\sqrt{a_{i}^{2}+b_{i}^{2}} / 2$.

An averaging over orientations and positions has been considered in the framework of ellipseellipse collisions. In this paper it is assumed that for cluster-cluster collisions the large number of collision events or Monte Carlo runs leads to a behaviour close to the ellipse-ellipse collision.

The figure 11 represents the gyration radius of the clusters as a function of the number of primary particles per aggregate for the collisions ellipse-ellipse without interpenetration $(B=$ $0)$, collisions ellipse-ellipse with interpenetration $(B=2)$ and collisions cluster-cluster. The selected (time) steps are 700, 1000, 1250 for each kind of collisions. It is observed that the agreement is very good between cluster-cluster and ellipse-ellipse $(B=2)$ collisions. The relation $R_{g}(N)$ for cluster-cluster collisions obeys:

$$
R_{g} / R_{1}=k N^{1 / D_{f}}=0.7 N^{1 / 1.5} \quad \text { as } \quad 3<N<8000
$$

Thus, the fractal dimension is equal to 1.5 .

However there exists a difference between the two kinds of collisions: in the case of clustercluster collisions, it appears very few ( $<1 \%$ of all clusters), but very large clusters. As a collision involving a large aggregate is more likely, larger and larger aggregates are produced. In our opinion, the main reasons of this behaviour are the use of the Monte Carlo algorithm with the kernel (Eq.36) and the microscopic nature of the collision act as well. This 
phenomenon cannot be ignored when studying aggregation at long time. However, experimental results show that the aggregates moving in a shear flow contain a small number of primary particles $\left(N<<10^{3}\right)$; this is due to the fragmentation of large and loose aggregates. Ellipse-ellipse collisions do not lead to rare and large aggregate.

Another difference concerns the elongation distributions (figures 12a-b). In the case of cluster-cluster collisions, the distribution has a tail for the large values of elongation. The distribution of elongation issued from the equivalent ellipses has no such characteristics. However, the peaks of the elongation distributions occur at the same elongation value which is smaller than 2 for the two kinds of collisions.

\section{Conclusion}

The inertia tensor of a particle is directly related to the gyration radius and the anisotropy factors, or the elongation. One may deduce the density or the volume fraction inside the particle by knowing the mass or number of primary particles. In our modelling these data are used to define an equivalent porous ellipsoid. From this, the variation of the morphological parameters during the collision event has been calculated. This result has been applied to the entire particle population to simulate the aggregation dynamics in the shear aggregation of disks in a two-dimensional space. The resulting aggregate, or ellipse, depends on the location of the contact point between colliding ellipses. It has been shown by different ways that the aggregates interpenetrate during the collision. The penetration length has been estimated. However the mechanism of interpenetration involved for fractal-like aggregates cannot be strictly applied to the equivalent ellipses in the current model. By using a weak definition of the fractality, one observes that the density of the particles follows a power law as fractal-like particles do. The deduced fractal dimension is within the range [1.3-1.5], the larger values corresponding to the deeper penetration. Hentschel and Meakin showed that the ballistic 
cluster-cluster collision leads to a fractal dimension value in the range [1.375-1.55] whereas, in this work, cluster-cluster collision in a shear flow leads to a value equal to 1.5. As a consequence, the ellipse-ellipse collisions with interpenetration show well the main features of the cluster-cluster collisions. This proves the relevance of this approach. The proposed modelling gives an important role to the anisotropy factor. It is observed that aggregation produces anisotropic particles. Most of particles have a value of elongation close to 2 in agreement with the literature. However, elongation distribution issued from cluster-cluster collisions is right-skewed unlike the one coming from ellipse-ellipse collision. Anisotropy factor is also a relevant parameter for fragmentation: real large aggregate in a real shear flow elongates and then breaks up at the most fragile part inside it.

This approach will be extended to the shear aggregation in a three-dimensional space. As expected from first investigations, the calculations related to the ellipsoid-ellipsoid collision are slightly more tedious and leads to time-consuming computations of the aggregation dynamics. However approximate method like the one developed for the two-dimensional case could notably improve this point.

\section{References}

[1] G.E. Schroeder-Turk, W. Mickel, S.C. Kapfer, F.M. Schaller, B. Breidenbach, D. Hug, K. Mecke, "Minkowski tensors of anisotropic spatial structure“,arXiv: 1009.2340v5 6 Aug 2013

[2] C.H. Arns, M.A. Knackstedt, K.R. Mecke, "Characterisation of irregular spatial structures by parallel sets and integral geometric measures", Colloids and Surfaces A: Physicochemical and Engineering Aspects 241 (2004) 351-372

[3] F.E. Torres, W.B. Russel, W.R. Schowalter, "Simulations of coagulation in viscous flows", J. of Colloid and Interface Science 145 (1991) 51-73 
[4] G.W. Mulholland, L. Zhou, M.R. Zachariah, W.R. Heinson, A. Chakrabarti, C.

Sorensen, "Light scattering shape diagnostics for nano-agglomerates", Aerosol Science and Technology 47 (2013) 520-529

[5] R. Sanchez, P. Bartlett, "Equilibrium cluster formation and gelation”, arXiv:condmat/0506566v1 22 Jun 2005

[6] A. Sikorski, "Structure of dense polymer systems confined to a slit", Acta Physica Polonica A 109 (2006) 133-141

[7] V. Blavatska, C. von Ferber, Yu. Holovatch, "Universal features of polymer shapes in crowded environments”, Physics Letters A 374 (2010) 2861-2864

[8] A.D. Melas, L. Isella, A.G. Konstandopoulos, Y. Drossinos, "Morphology and mobility of synthetic colloidal aggregates", J. of Colloid and Interface Science 417 (2014) 27-36

[9] J. Rudnick, G. Gaspari, “The asphericity of random walks”, J. Phys. A: Math. Gen. 19 (1986) L191-193

[10] J. Rudnick, G. Gaspari, “The shapes of random walks”, Science 237 (1987) 384-389

[11] G. Pranami, M.H. Lamm, R.D. Vigil, "Molecular dynamics simulation of fractal aggregate diffusion”, Physical Review E82 051402 (2010)

[12] L. Landau et E. Lifchitz, Mécanique, Editions MIR, Moscou, 1969

[13] A. Mongruel, "Etude expérimentale de suspensions de particules anisotropes en écoulement élongationnel", thèse de doctorat, Ecole Nationale des Ponts et Chaussées, Paris - Marne la vallée, 1994

[14] A.A. Potanin, "On the mechanism of aggregation in the shear flow of suspensions", J. of Colloid and Interface Sci. 145 (1991) 140-157

[15] M.S. Ingber, L.A. Mondy “A numerical study of three-dimensional Jeffery orbits in shear flow", J. of Rheology 38 (1994) 1829 
[16] E.J. Hinch, L.G. Leal, "Rotation of small non-axisymmetric particles in a simple shear flow", J. of Fluid Mechanics 92 (1979) 591-608

[17] C.J. Lin, K.J. Lee, N.F. Sather, "Slow motion of two spheres in a shear flow”, J. of Fluid Mechanics 43 (1970) 35-47

[18] G.K. Batchelor, J.T. Green, “The hydrodynamic interaction of two small freelymoving spheres in a linear flow field", J. of Fluid Mechanics 56 (1972) 375-400

[19] D.J. Jeffrey, Y. Onishi, "Calculation of the resistance and mobility functions for two unequal rigid spheres in low Reynolds number flow”, J. of Fluid Mechanics 139 (1984) $261-290$

[20] M. Smoluchowski, "Versuch einer mathematischen Theorie der Koagulationskinetik kolloider Lösungen“, Z. Physik. Chem. 92 (1917) 129-168

[21] M. Smith, T. Matsoukas, "Constant-number Monte Carlo simulation of population balances", Chemical Engineering Science 53 (1998) 1777-?

[22] M. Elimelech, J. Gregory, X. Jia, R. Williams, "Particle deposition \& aggregation", Butterworth-Heinemann, 1995

[23] H.G.E. Hentschel, "The structure and fractal dimension of cluster-cluster aggregates" in "Kinetics of aggregation and gelation" edited by F. Family and D.P. Landau, NorthHolland, 1984

[24] P. Meakin, "Off lattice simulations of cluster-cluster aggregation in dimensions 2-6", Physics letters 107A (1985) 269-272

[25] S. Veerapaneni, M.R. Wiesner, "Hydrodynamics of fractal aggregates with radially varying permeability”, J. of Colloid and Interface Science 177 (1996) 45-57

[26] G. Neale, N. Epstein, W. Nader, "Creeping flow relative to permeable sphere”, Chemical Engineering Science 28 (1973) 1865-1874 
[27] A.S. Sangani, A. Acrivos, "Slow flow past periodic arrays of cylinders with application to heat transfer", International Journal Multiphase Flow 8 (1982) 193-206 


\section{List of figures}

Figure 1: collision between two equivalent ellipsoids

Figure 2: collision between two objects: the object 1 with a given orientation collides the object 2 at two different points. Any point on the surface of the object 2 may be a contact point with object 1 .

Figure 3: shear aggregation; collision

Figure 4: the four configurations used for the approximate inertia tensor.

Figure 5: elongation $\lambda=a / b$ as a function of the number of primary particles into the aggregate; hierarchical aggregation

Figure 6: density $\phi$ as a function of the number of primary particles into the aggregate; hierarchical aggregation. Log is the common logarithm.

Figure 7: distribution in number $\mathrm{N}$ of primary particles per aggregate; from left to right: black $t=0.86(500)$; red $t=3.19$ (1000), green $t=26.8$ (2000), blue $t=201.5$ (3000), yellow $t=550$ (3500), black $\mathrm{t}=1500$ (4000), green $\mathrm{t}=1.1110^{4}$ (5000). Calculated from the exact method (dashed line) and the approximate method (solid line). The value within parentheses is the number of steps (MCS).

Figure 8: elongation distribution (as the ratio of the two semi-axes); from left to right: green $\mathrm{t}=26.8$, blue $\mathrm{t}=201.5$, yellow $\mathrm{t}=550$, black $\mathrm{t}=1500$, red $\mathrm{t}=1.1110^{4}$. Approximate Method. Figure 9: density as a function of the number $\mathrm{N}$ of primary particles; black $\mathrm{t}=0.86$; red $\mathrm{t}=3.19$, green $\mathrm{t}=26.8$, blue $\mathrm{t}=201.5$, yellow $\mathrm{t}=550$, black $\mathrm{t}=1500$, green $\mathrm{t}=1.1110^{4}$. The straight line $(\log -\log )$ corresponds to the equation $\phi=1.1 N^{-0.53}$. Collision ellipse-ellipse. Exact Method. Figure 10a: elongation distribution (as the ratio of the two semi-axes); from left to right: green $\mathrm{t}=26.8$, blue $\mathrm{t}=201.5$, yellow $\mathrm{t}=550$, black $\mathrm{t}=1500$, red $\mathrm{t}=1.1110^{4}$. Exact method. 
Figure 10b: elongation distribution (as the ratio of the two principal inertia moments); from left to right: green $\mathrm{t}=26.8$, blue $\mathrm{t}=201.5$, yellow $\mathrm{t}=550$, black $\mathrm{t}=1500$, red $\mathrm{t}=1.1110^{4}$. Exact method.

Figure 11: gyration radius against the number $\mathrm{N}$ of primary particle per aggregate. green: collisions ellipse-ellipse without interpenetration $(B=0)$; red: collisions ellipse-ellipse with interpenetration $(B=2)$; blue: collisions cluster-cluster.

Figure 12a: elongation distribution $(\mathrm{N}>2)$ for three time steps: red: 700 ; green : 1000 ; blue: 1250 cluster-cluster collision Figure 12b: elongation distribution $(\mathrm{N}>2)$ for three time steps: red: 700 ; green: 1000 ; blue: 1250 ellipse-ellipse collision 


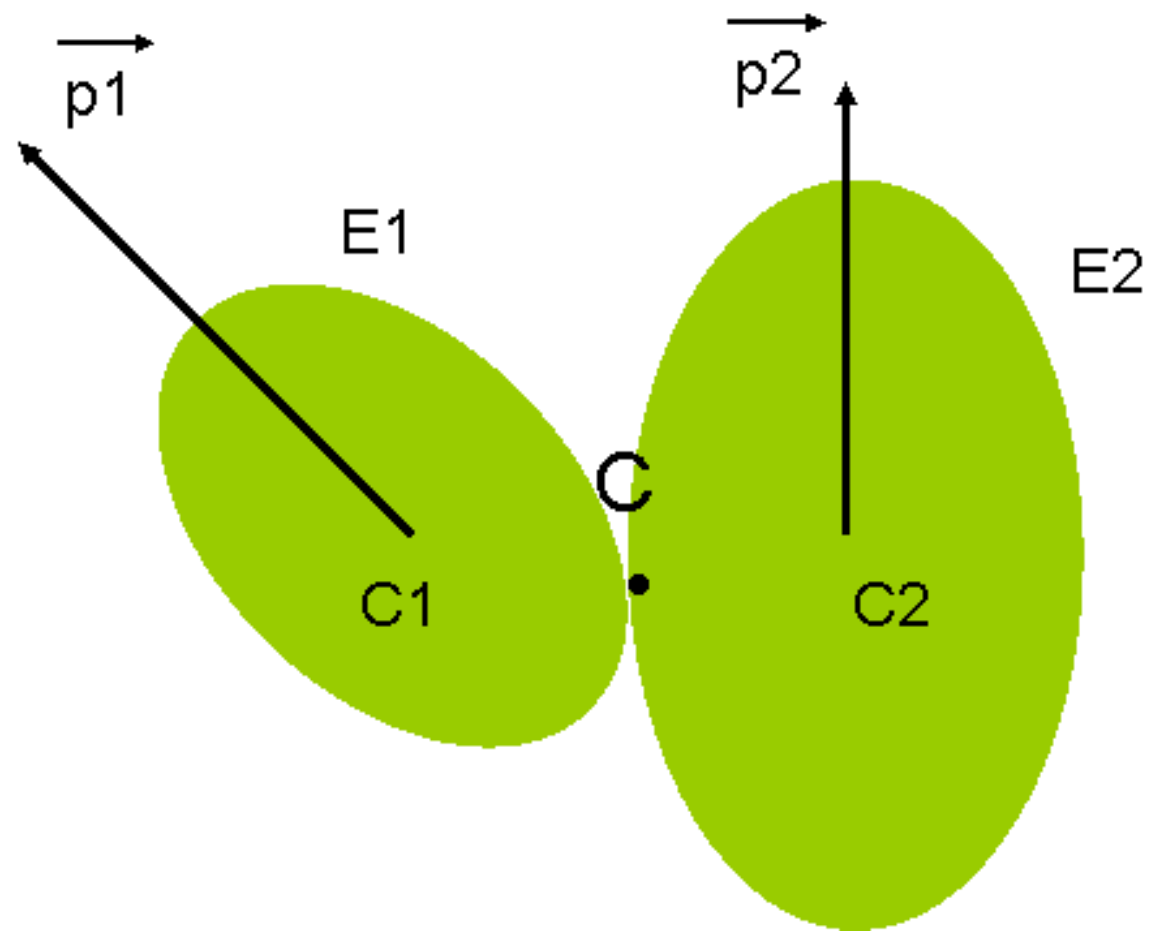

Figure 1: collision between two equivalent ellipsoids 


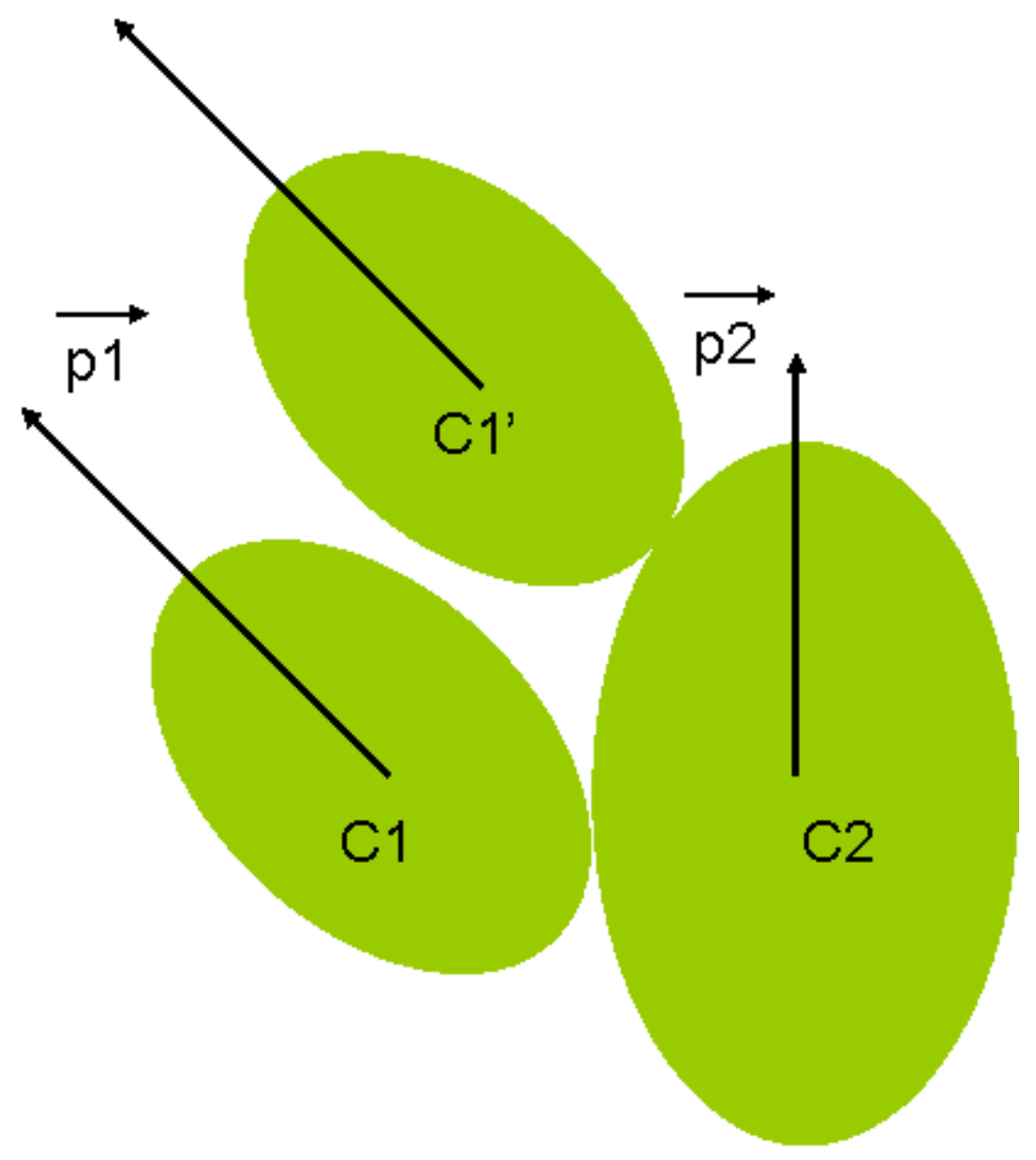

Figure 2: collision between two objects: the object 1 with a given orientation collides the object 2 at two different points. Any point on the surface of the object 2 may be a contact point with object 1 . 

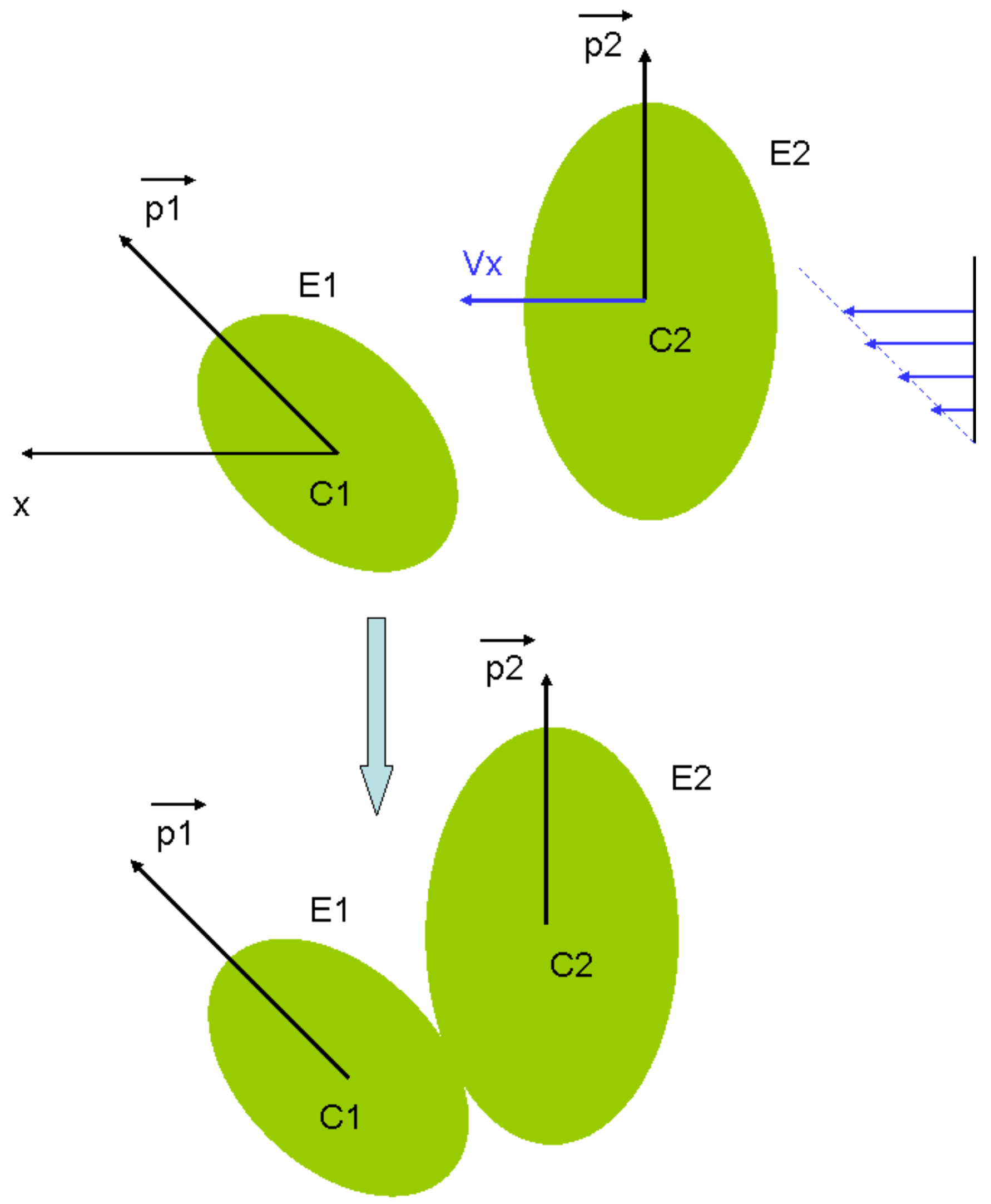

Figure 3: shear aggregation; collision 


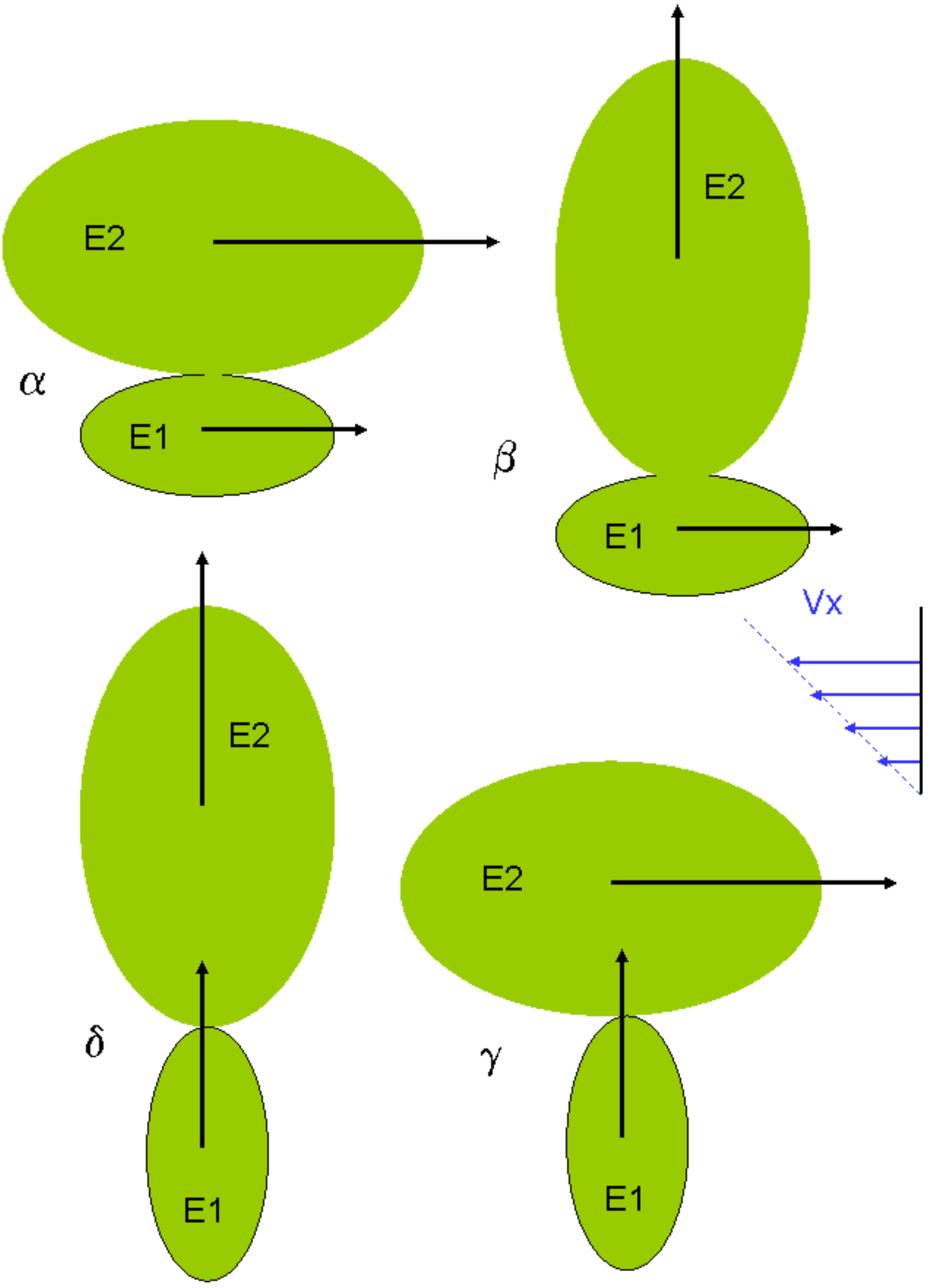

Figure 4: The four configurations used for the approximate inertia tensor. 


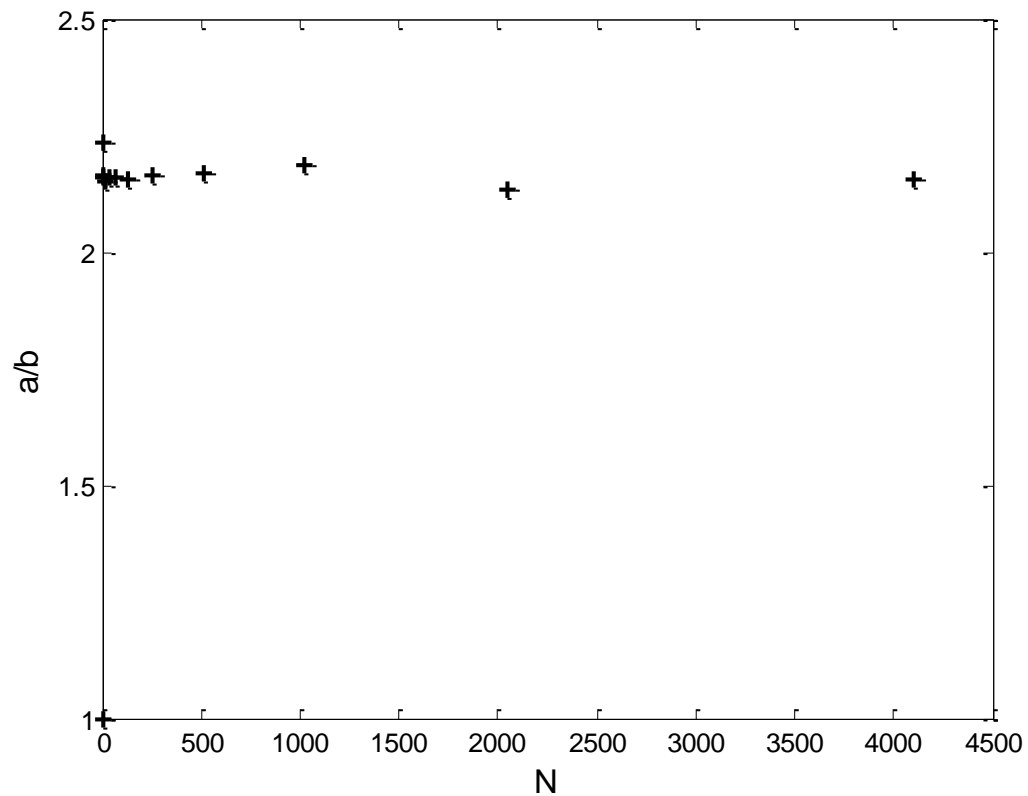

Figure 5: elongation $\lambda=a / b$ as a function of the number of primary particles into the aggregate; hierarchical aggregation 


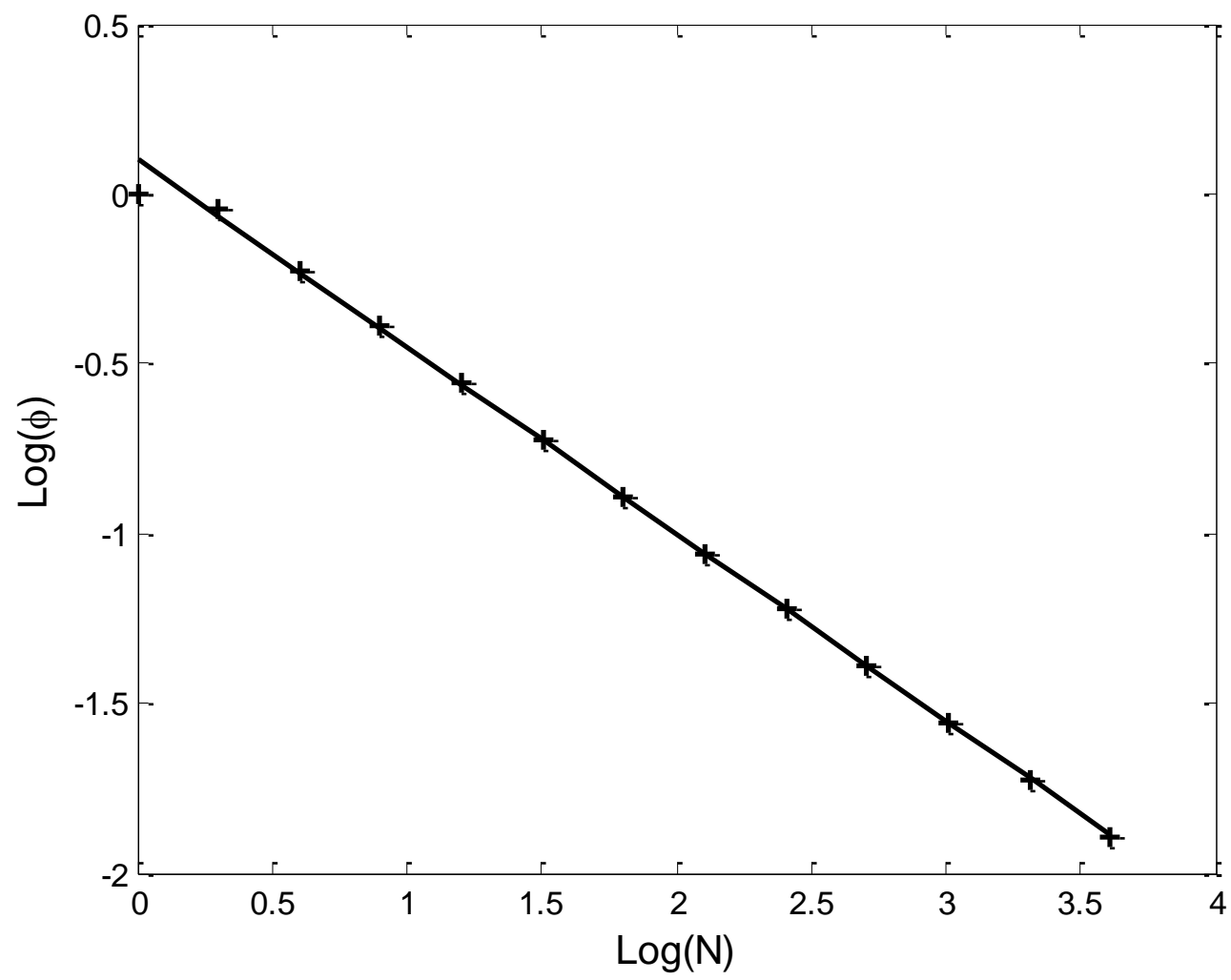

Figure 6: density $\phi$ as a function of the number $\mathrm{N}$ of primary particles into the aggregate; hierarchical aggregation. Log is the common logarithm. 


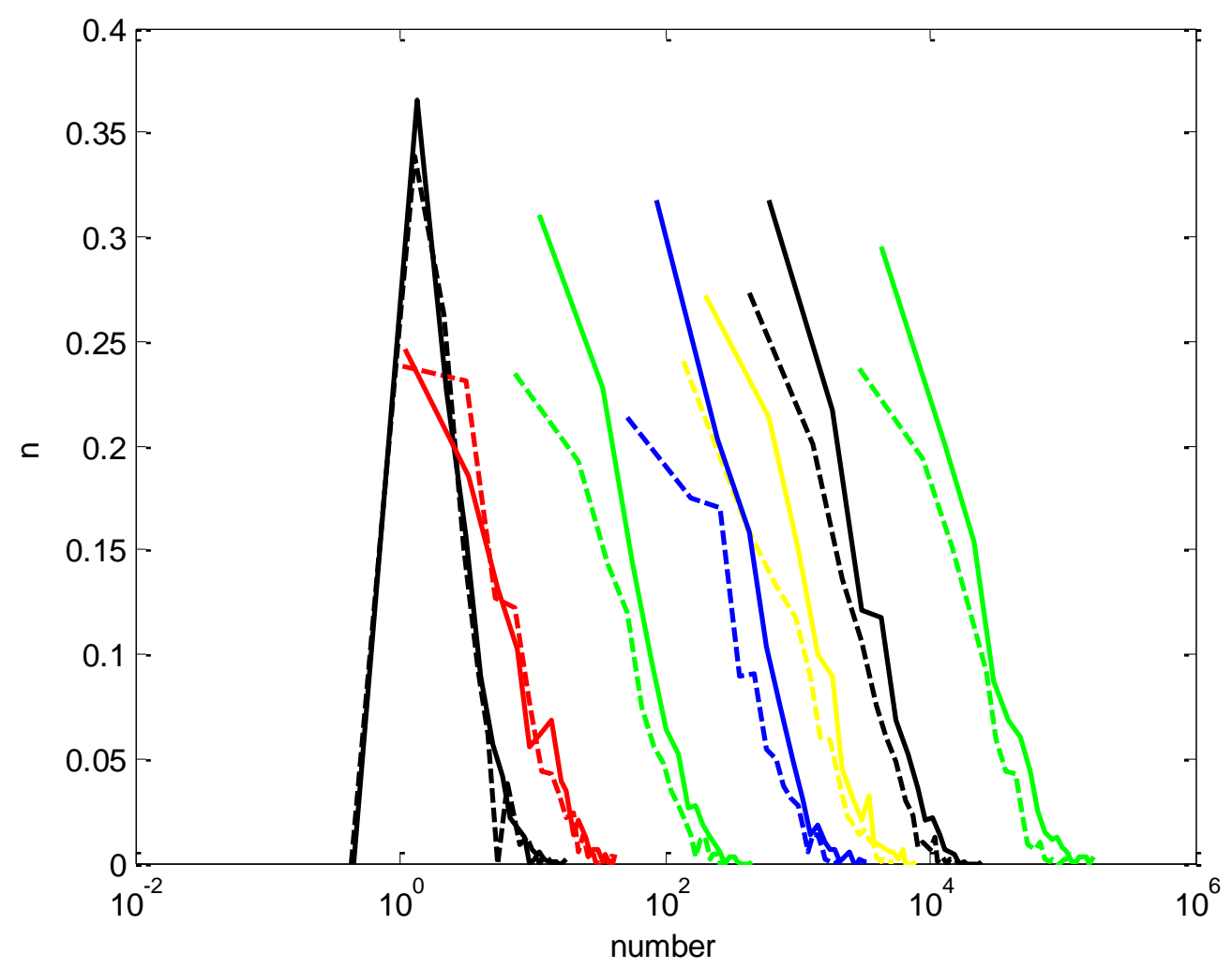

Figure 7: distribution in number $\mathrm{N}$ of primary particles per aggregate; from left to right: black $t=0.86(500)$; red $t=3.19$ (1000), green $t=26.8$ (2000), blue $t=201.5(3000)$, yellow $t=550$ (3500), black $\mathrm{t}=1500$ (4000), green $\mathrm{t}=1.1110^{4}$ (5000). Calculated from the exact method (dashed line) and the approximate method (solid line). The value within parentheses is the number of steps (MCS). 




Figure 8: elongation distribution (as the ratio of the two semi-axes); from left to right: green $\mathrm{t}=26.8$, blue $\mathrm{t}=201.5$, yellow $\mathrm{t}=550$, black $\mathrm{t}=1500$, red $\mathrm{t}=1.1110^{4}$. Approximate Method. 


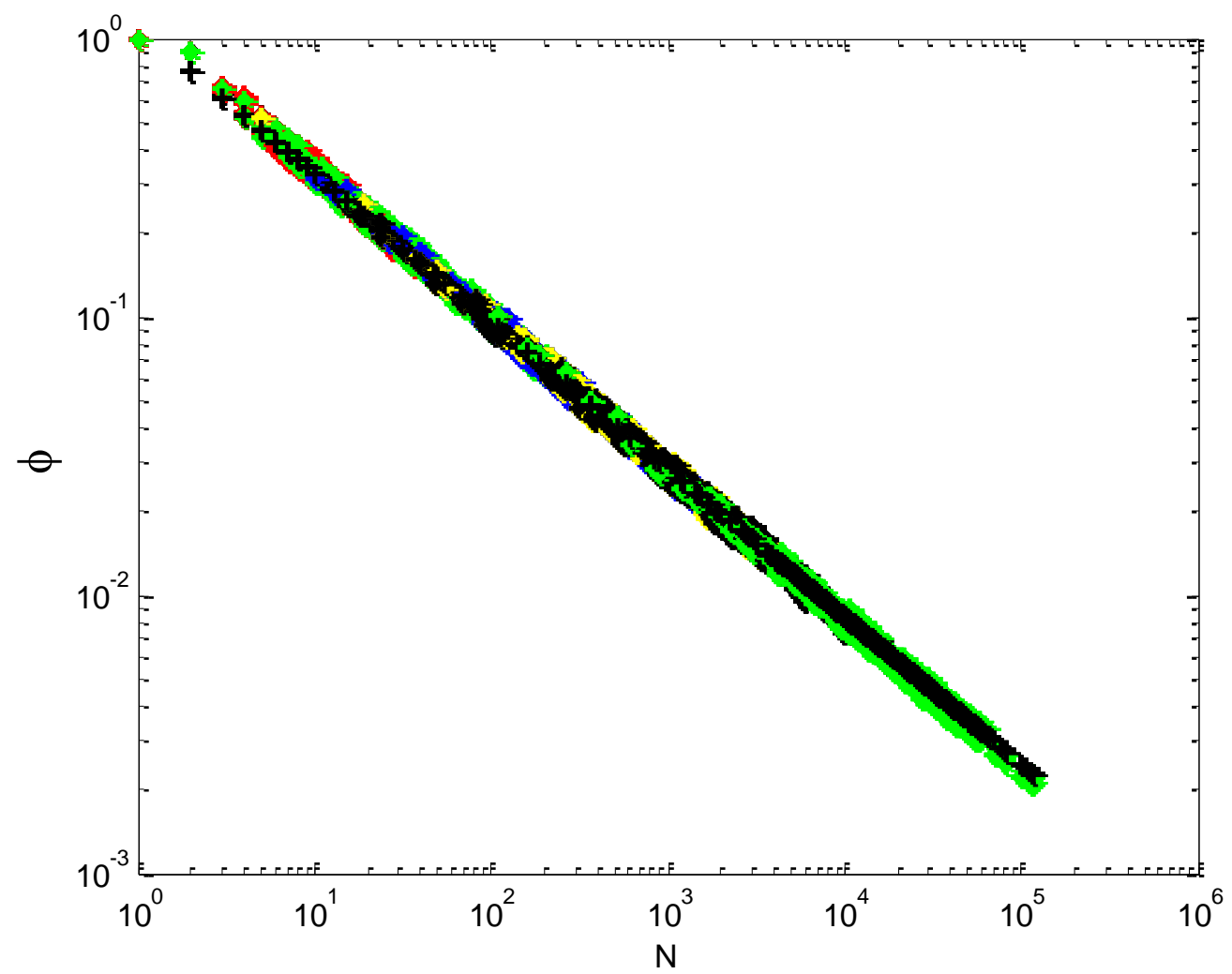

Figure 9: density as a function of the number $\mathrm{N}$ of primary particles; black $\mathrm{t}=0.86$; red $\mathrm{t}=3.19$, green $\mathrm{t}=26.8$, blue $\mathrm{t}=201.5$, yellow $\mathrm{t}=550$, black $\mathrm{t}=1500$, green $\mathrm{t}=1.1110^{4}$. The straight line $(\log -\log )$ corresponds to the equation $\phi=1.1 N^{-0.53}$. Collision ellipse-ellipse. Exact Method. 




Figure 10a: elongation distribution (as the ratio of the two semi-axes); from left to right: green $\mathrm{t}=26.8$, blue $\mathrm{t}=201.5$, yellow $\mathrm{t}=550$, black $\mathrm{t}=1500$, red $\mathrm{t}=1.1110^{4}$. Exact method. 


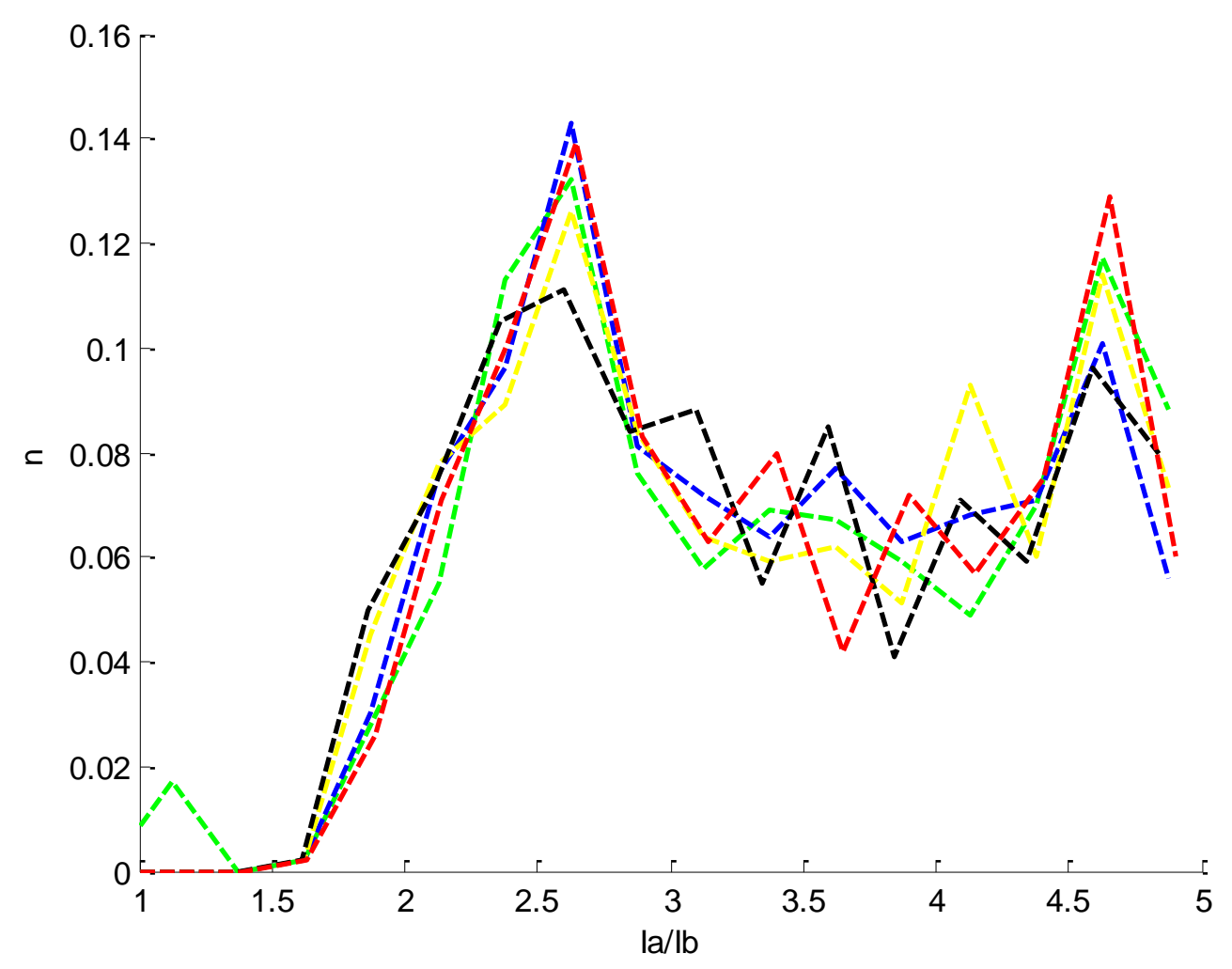

Figure 10b: elongation distribution (as the ratio of the two principal inertia moments); from left to right: green $\mathrm{t}=26.8$, blue $\mathrm{t}=201.5$, yellow $\mathrm{t}=550$, black $\mathrm{t}=1500$, red $\mathrm{t}=1.1110^{4}$. Exact method. 


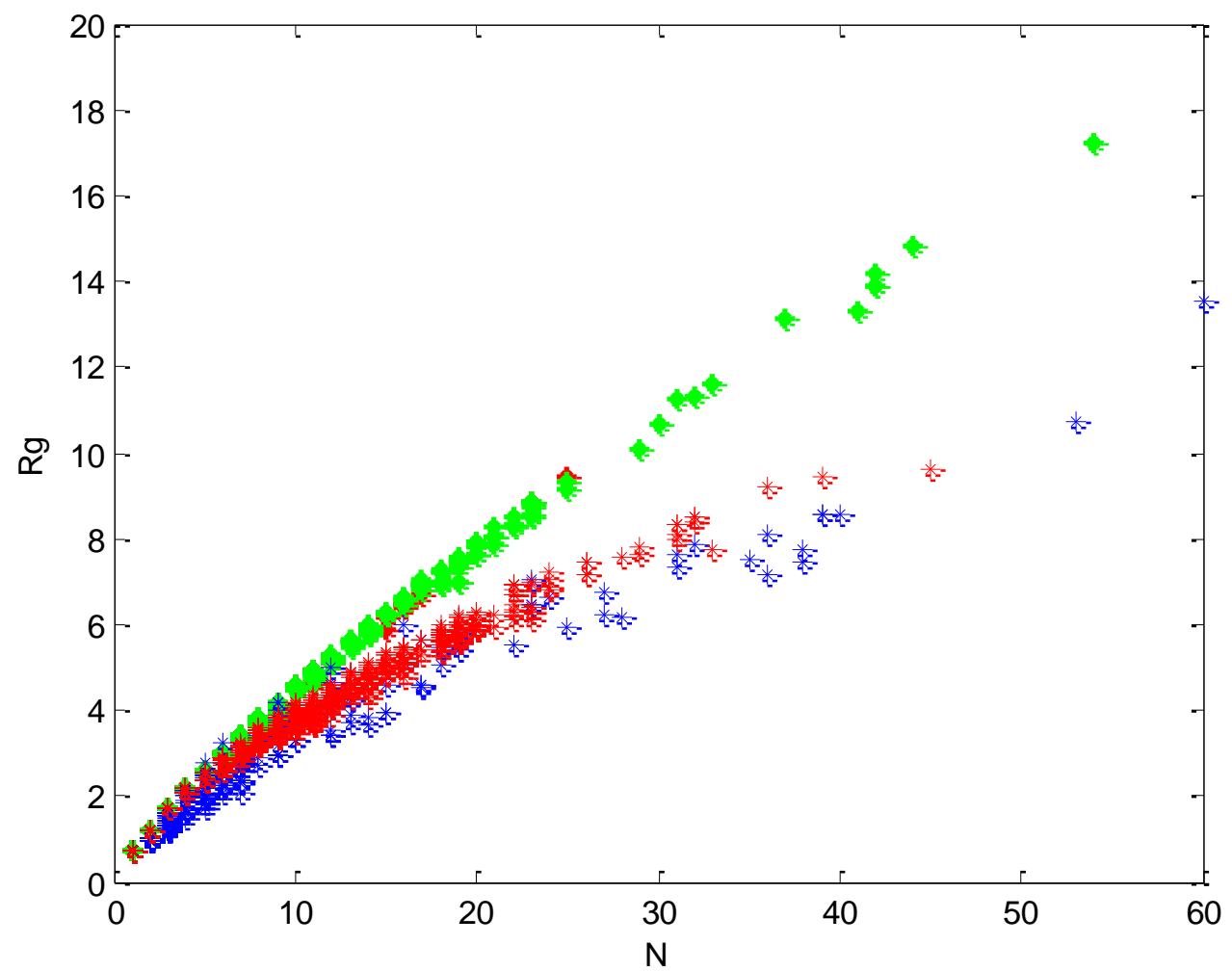

Figure 11: gyration radius against the number $\mathrm{N}$ of primary particle per aggregate. green: collisions ellipse-ellipse without interpenetration $(B=0)$; red: collisions ellipse-ellipse with interpenetration $(B=2)$; blue: collisions cluster-cluster. 


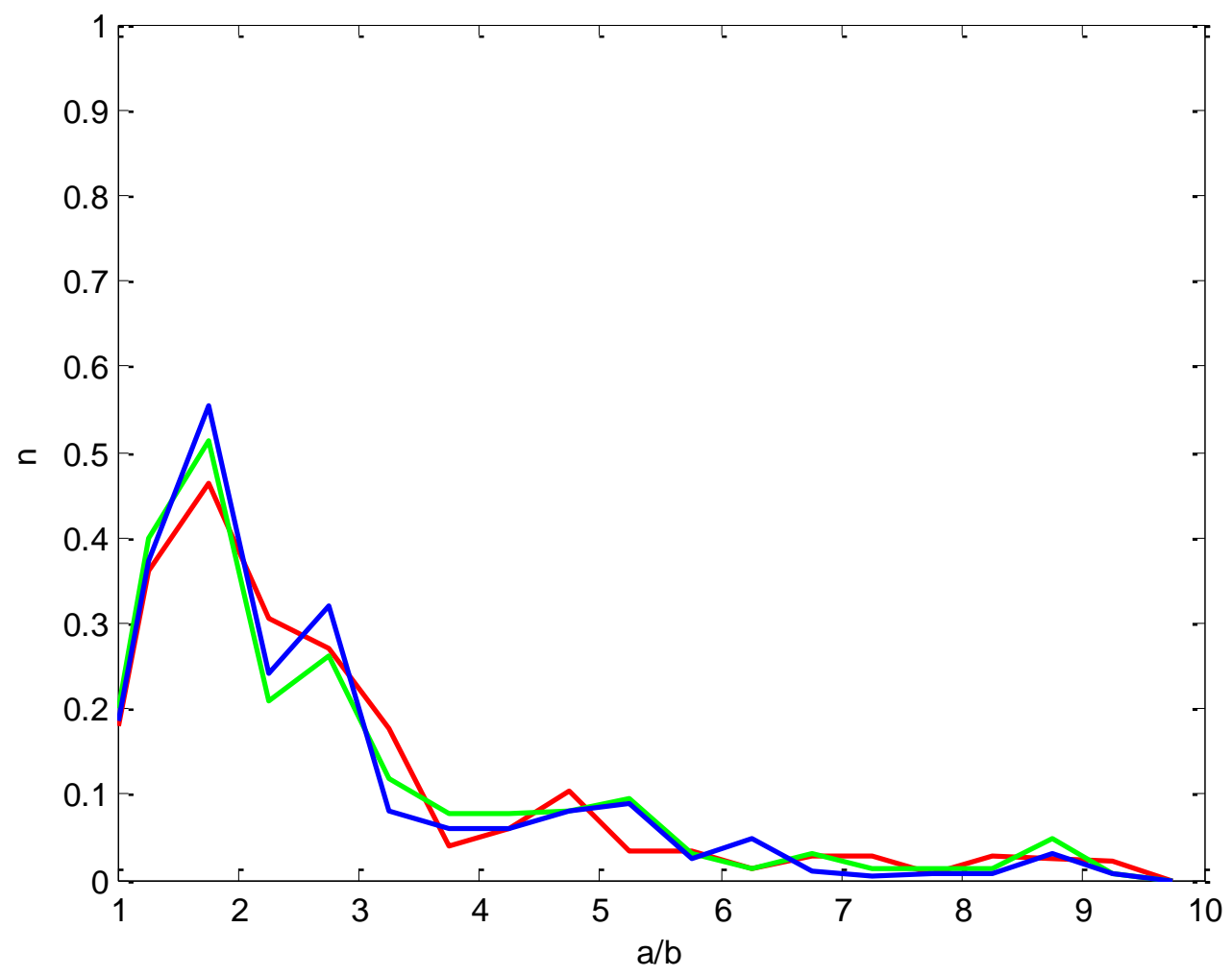

Figure 12a: elongation distribution $(\mathrm{N}>2)$ for three time steps:

red : 700 ; green : 1000 ; blue : 1250 cluster-cluster collision 




Figure 12b: elongation distribution $(\mathrm{N}>2)$ for three time steps:

red: 700 ; green: 1000 ; blue: 1250 ellipse-ellipse collision 


\section{List of tables}

Table 1: parameters of the function density-number $\phi=k N^{n}=k N^{(D f-2) / D f}$. Effect of the penetration parameter $B$; Approximate method.

Table 2: parameters of the function density-number $\phi=k N^{n}=k N^{(D f-2) / D f}$. Effect of the penetration parameter $B$; Exact method. 


\begin{tabular}{|c|c|c|c|}
\hline B & $\mathrm{k}$ & $\mathrm{n}$ & Df \\
\hline 0 & 1.1 & 0.59 & 1.26 \\
\hline 1 & $\begin{array}{ll}1 & \mathrm{~N}<500 \\
1.8 & \mathrm{~N}>500\end{array}$ & $\begin{array}{l}0.46 \\
0.56\end{array}$ & $\begin{array}{l}1.37 \\
1.28\end{array}$ \\
\hline 2 & $\begin{array}{ll}0.9 & N<500 \\
3.5 & N>500\end{array}$ & $\begin{array}{l}0.36 \\
0.56\end{array}$ & $\begin{array}{l}1.47 \\
1.28\end{array}$ \\
\hline 3 & $\begin{array}{ll}0.9 & N<500 \\
5 & N>500\end{array}$ & $\begin{array}{l}0.3 \\
0.56\end{array}$ & $\begin{array}{l}1.54 \\
1.28\end{array}$ \\
\hline
\end{tabular}

Table 1: parameters of the function density-number $\phi=k N^{n}=k N^{(D f-2) / D f}$. Effect of the penetration parameter $B$; Approximate method. 


\begin{tabular}{|c|c|c|c|}
\hline B & $\mathrm{k}$ & $\mathrm{n}$ & Df \\
\hline 0 & 1.1 & 0.53 & 1.31 \\
\hline 1 & $\begin{array}{ll}1.1 & \mathrm{~N}<150 \\
1.7 & \mathrm{~N}>150\end{array}$ & $\begin{array}{l}0.46 \\
0.52\end{array}$ & $\begin{array}{l}1.37 \\
1.32\end{array}$ \\
\hline 2 & $\begin{array}{ll}1.1 & N<350 \\
2.1 & N>350\end{array}$ & $\begin{array}{l}0.41 \\
0.51\end{array}$ & $\begin{array}{l}1.42 \\
1.33\end{array}$ \\
\hline 3 & & & \\
\hline
\end{tabular}

Table 2: parameters of the function density-number $\phi=k N^{n}=k N^{(D f-2) / D f}$. Effect of the penetration parameter $B$; Exact method. 